Diagnostic Images

\title{
A red herring with black disseminated disease
}

\author{
Presented by M.A. Al-Kutoubi and L. Kreel
}

Radiology Department, St Mary's Hospital, Praed Street, Paddington, London W2, UK

\section{The patient}

A woman of 79 , known to have had an excision of skin nodules 9 months previously, was admitted for recurrent lesions at the operative site. Three years later there was further local recurrence. On examination, a large left abdominal mass was noted.

\section{Investigations}

Investigations included a chest film, a radiograph of the right femur, abdominal sonography, an isotope bone scan and abdominal computed tomography (CT).
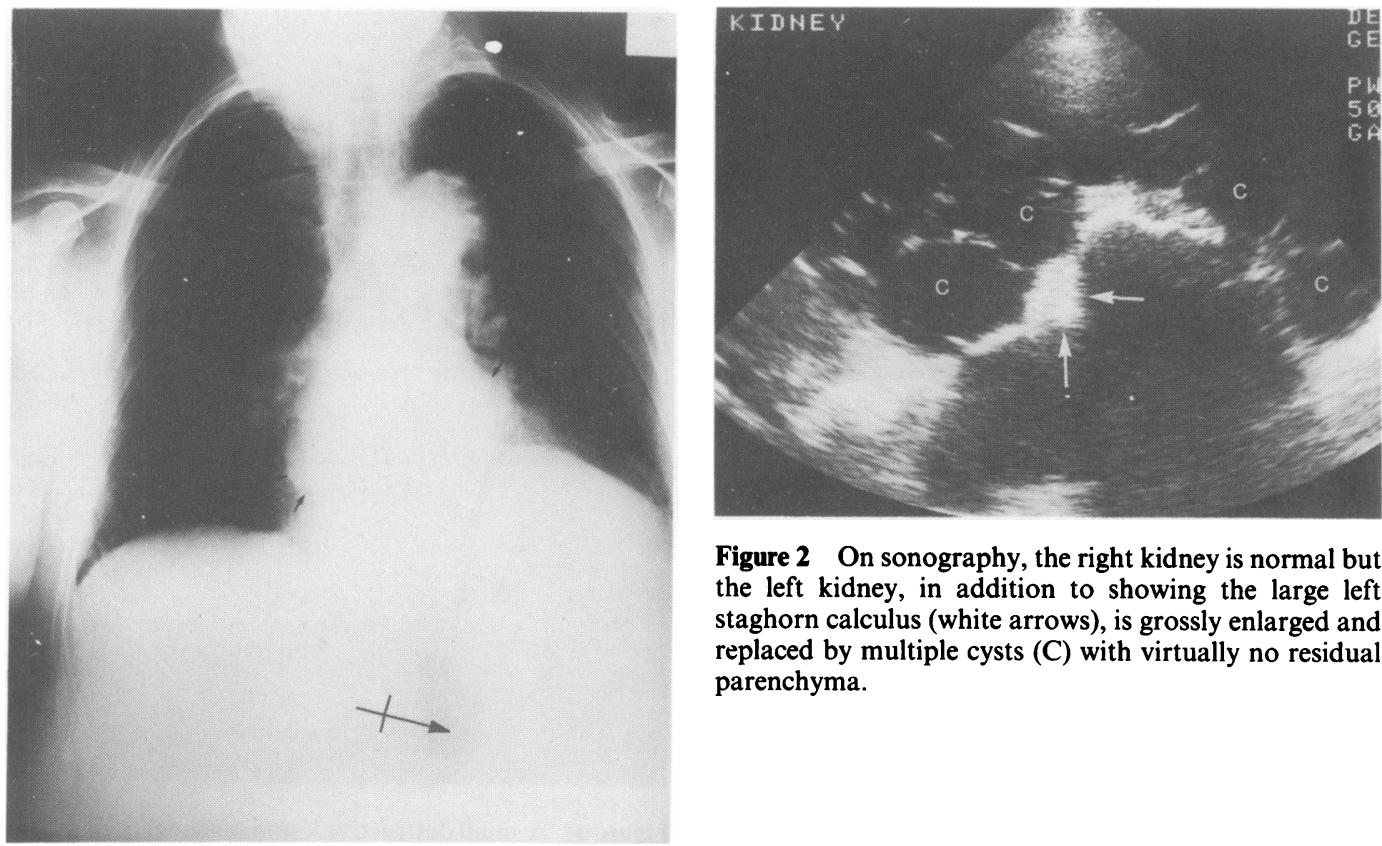

Figure 2 On sonography, the right kidney is normal but the left kidney, in addition to showing the large left staghorn calculus (white arrows), is grossly enlarged and replaced by multiple cysts $(C)$ with virtually no residual parenchyma.

Figure 1 On the chest film the left hemidiaphragm is markedly raised, the cardiac contour is enlarged mainly to the left, the aortic knuckle and descending aorta are prominent and the left atrium is seen as a double density (arrows). A large left staghorn calculus is just visible at the position of the crossed arrows. 
a

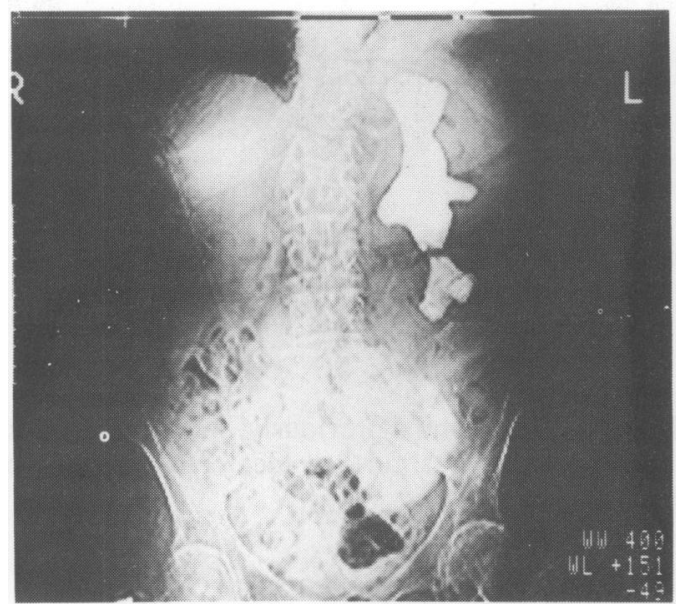

b

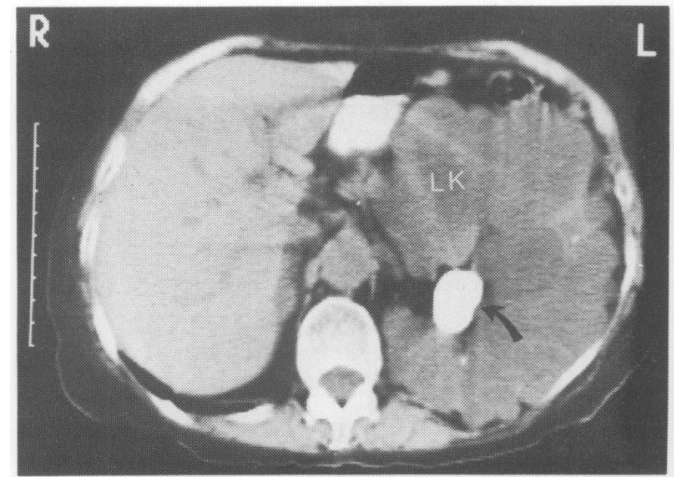

Figure 3 a, b, The scanogram of the CT clearly demonstrates the large staghorn calculus, the raised left hemidiaphragm and the large left kidney. CT sections confirm a multicystic left kidney (LK) displacing the stomach anteriorly and to the right with a large hilar calculus (arrow). The right kidney is normal. While no metastases were seen in the liver, spleen or lymph nodes, the right adrenal is enlarged suggesting a secondary deposit.

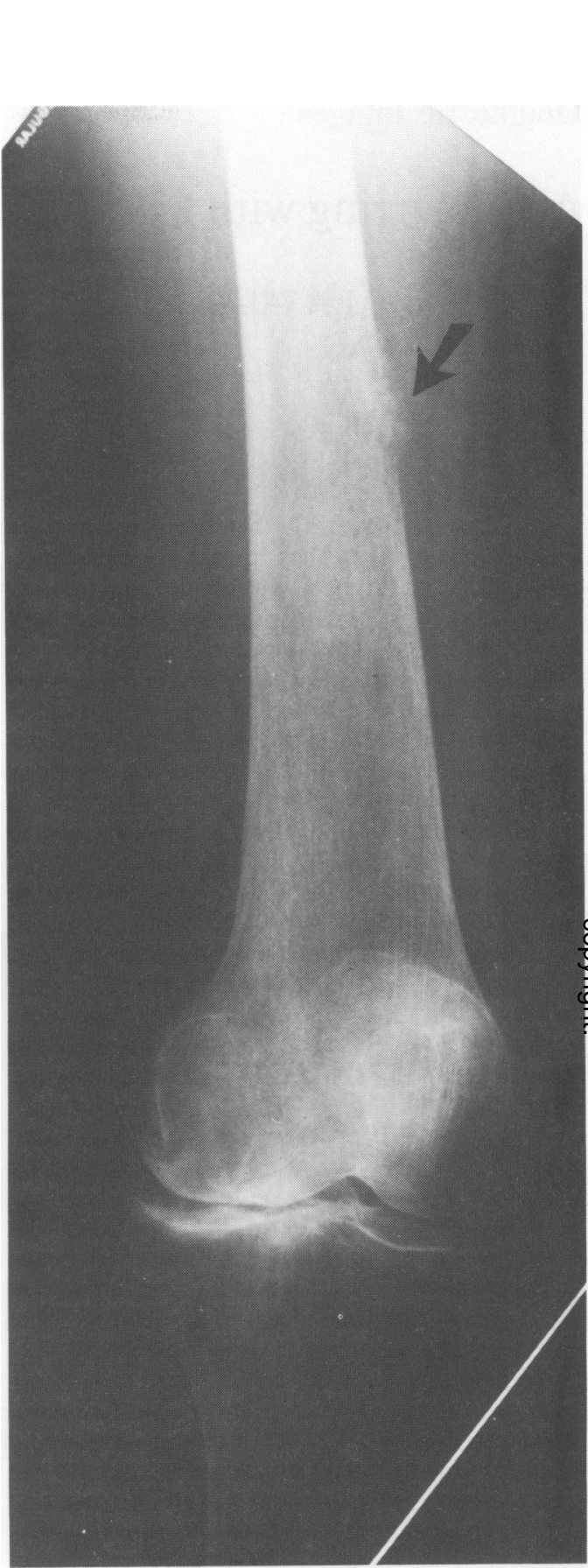

Figure 4 A small destructive lesion is shown in the dista third of the right femur (arrow) with elevation of the periosteum indicating a bone metastasis. 
a

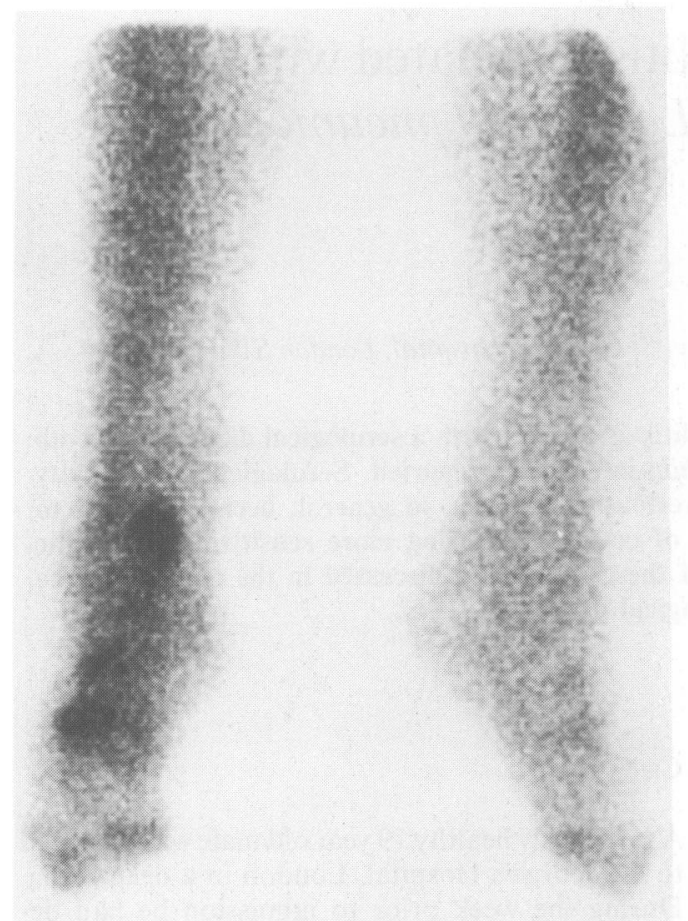

b

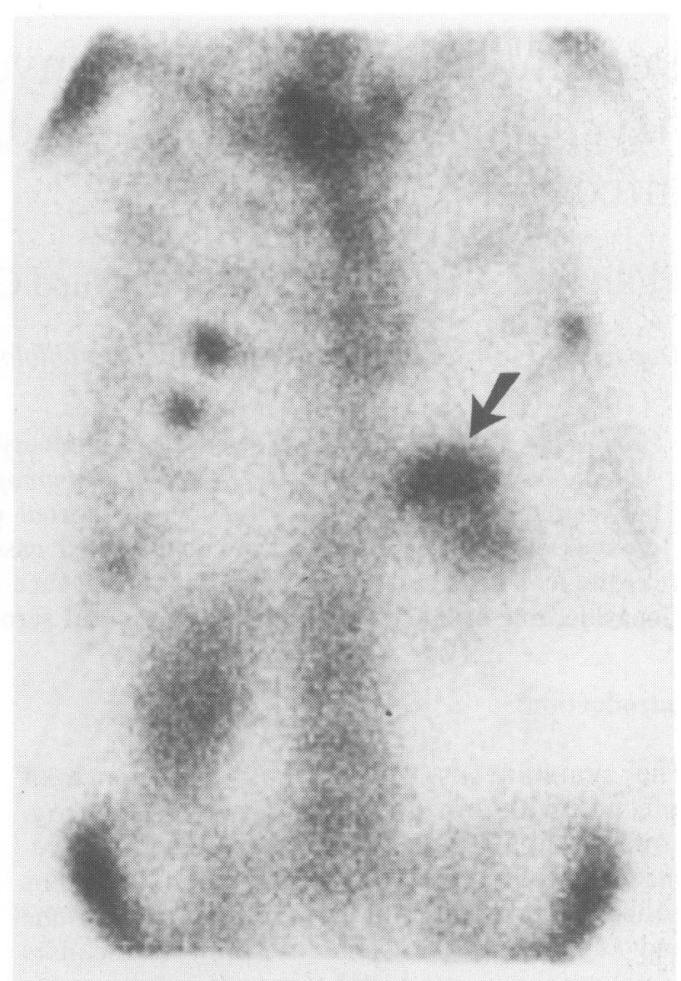

Figure 5 a, b Isotope bone scan showing multiple areas of increased uptake (ribs, skull, femur, tibia, tarsus) and also uptake in the left staghorn calculus (arrow).

\section{Comment}

A raised left hemidiaphragm also elevates the heart and increases the cardiothoracic ratio. The double density of the left atrium can occur with longstanding left ventricular enlargement but unlike mitral valve disease, does not produce enlargement of the left atrial appendage.

Phrenic nerve palsy, pulmonary collapse and intra-abdominal masses can cause a raised hemidiaphragm. The staghorn calculus would suggest a possible gross hydronephrosis but sonography was conclusive in showing a unilateral multicystic kidney, confirmed by CT. The left abdominal mass was not caused by the recurrent melanoma that subsequently produced multiple bone metastases. The destructive lesion in the femur in the context of a known primary malignancy probably indicates a metastasis but de novo may be due to a primary bone tumour or infection. However, this patient does illustrate how much more sensitive isotope bone scanning is than plain film radiography which did not show the other bone lesions. The intense isotope uptake in the left kidney in the absence of a known renal calculus could be due to a hydronephrosis or a tumour. 